\title{
Constitutional and somatic methylation status of DMRH19 and KvDMR in Wilms tumor patients
}

Leila C.A. Cardoso ${ }^{1,3}$, Jair A. Tenorio Castaño ${ }^{2}$, Hanna S. Pereira ${ }^{3}$, Maria Angélica de F.D. Lima ${ }^{3,7}$, Anna Cláudia E. dos Santos ${ }^{1}$, Paulo S. de Faria ${ }^{4}$, Sima Ferman $^{5}$, Héctor N. Seuánez ${ }^{1,3}$, Julián B. Nevado ${ }^{2}$, José Carlos Cabral de Almeida ${ }^{6}$, Pablo Lapunzina ${ }^{2}$ and Fernando R. Vargas ${ }^{1,3,7}$

${ }^{1}$ Departamento de Genética, Universidade Federal do Rio de Janeiro, Rio de Janeiro, RJ, Brazil.

${ }^{2}$ Instituto de Genética Médica y Molecular, Hospital Universitario La Paz, Centro de Investigación Biomédica en Red de Enfermedades Raras, Madrid, Spain.

${ }^{3}$ Programa de Genética, Instituto Nacional de Câncer, Rio de Janeiro, RJ, Brazil.

${ }^{4}$ Divisão de Patologia, Instituto Nacional de Câncer, Rio de Janeiro, RJ, Brazil.

${ }^{5}$ Serviço de Oncologia Pediátrica, Instituto Nacional de Câncer, Rio de Janeiro, RJ, Brazil.

${ }^{6}$ Departamento de Genética Médica, Instituto Nacional de Saúde da Mulher, da Criança e do Adolescente Fernandes Figueira, Fundação Oswaldo Cruz, Rio de Janeiro, RJ, Brazil.

${ }^{7}$ Departamento de Genética e Biologia Molecular, Universidade Federal do Estado do Rio de Janeiro, Rio de Janeiro, RJ, Brazil.

\begin{abstract}
The most frequent epigenetic alterations in Wilms tumor (WT) occur at WT2, assigned to 11p15. WT2 consists of two domains: telomeric domain 1 (DMRH19) that contains the IGF2 gene and an imprinted maternally expressed transcript (H19) and centromeric domain 2 (KvDMR) that contains the genes KCNQ1, KCNQ1OT1 and CDKN1C. In this work, we used pyrosequencing and MS-MLPA to compare the methylation patterns of DMRH19/KvDMR in blood and tumor samples from 40 WT patients. Normal constitutional KvDMR methylation indicated that most of the epigenetic alterations in WT occur at DMRH19. Constitutional DMRH19 hypermethylation (HM DMRH19) was observed in two patients with Beckwith-Wiedemann syndrome. Pyrosequencing and MS-MLPA showed HM DMRH19 in 28/34 tumor samples: 16/34 with isolated HM DMRH19 and 12/34 with concomitant HM DMRH19 and KvDMR hypomethylation, indicating paternal uniparental disomy. With the exception of one blood sample, the MS-MLPA and pyrosequencing findings were concordant. Diffuse or focal anaplasia was present in five tumor samples and was associated with isolated somatic HM DMRH19 in four of them. Constitutional 11p15 methylation abnormalities were present in $5 \%$ of the samples and somatic abnormalities in the majority of tumors. Combined analysis of DMRH19/KvDMR by pyrosequencing and MS-MLPA is beneficial for characterizing epigenetic anomalies in WT, and MS-MLPA is useful and reliable for estimation of DNA methylation in a clinical setting.
\end{abstract}

Keywords: epigenetic, histopathology, methylation, MS-MLPA, pyrosequencing.

Received: May 30, 2012; Accepted: July 24, 2012.

\section{Introduction}

Wilms tumor (WT) is the most frequent extra-cranial solid cancer in childhood, accounting for $6-7 \%$ of all childhood neoplasias (Miller et al., 1995). In 80\% of cases, WT is diagnosed in children less than five years old. In Brazil, the age-adjusted incidence for WT in children up to 14 years old varies from five to 18 cases per million (De Camargo et al., 2011). Bilateral presentation is observed in

Send correspondence to Fernando Regla Vargas. Programa de Genética, Instituto Nacional de Câncer, Rua André Cavalcanti 37, 4ำ andar, 20231-050 Rio de Janeiro, RJ, Brazil. E-mail address: fvargas@inca.gov.br.
$5-10 \%$ of affected individuals (Dome and Huff, 2011). In most cases, WT is present as a sporadic, isolated disorder, although it may sometimes be a component of complex, congenital clinical entities such as WAGR (Wilms, Aniridia, Genitourinary congenital anomalies, Retarded development) syndrome, Denys-Drash syndrome (DDS: male pseudohermaphroditism, renal mesangial sclerosis) and Beckwith-Wiedemann syndrome (BWS: overgrowth, omphalocele and hemihypertrophy), among others (Scott et al., 2008a; Nakamura and Ritchey, 2010).

The most frequent epigenetic alterations in WT occur at the WT2 region in $11 \mathrm{p} 15$, secondary to either imprinting 
center mutations that result in isolated epigenetic defects or paternal uniparental disomies (UPD) (Scott et al., 2008b). WT2 consists of two domains that contain several genes: the telomeric domain 1 contains the insulin-like growth factor 2 (IGF2) gene and an imprinted maternally expressed transcript (H19), while the centromeric domain 2 contains the potassium voltage-gated channel gene (KCNQ1), the $K C N Q 1$ opposite strand/antisense transcript 1 gene $($ KCNQ1OT1) and the dependent kinase inhibitor $1 \mathrm{C}$ gene (CDKN1C) (Smith et al., 2007; Scott et al., 2008a).

In domain $1, I G F 2$ is normally expressed only by the paternal allele, in contrast to $H 19$ that transcribes an untranslated messenger RNA that is only maternally expressed. The expression of these genes is regulated by the differentially methylated region H19 (DMRH19) located upstream of the $H 19$ promoter; this region is normally methylated only in the paternal allele. Abnormal methylation of DMRH19 leads to loss of IGF2 imprinting control and abnormal IGF2 expression by the maternal allele; expression of the latter gene is normally inhibited by ligation to the zinc-finger CCCTC-binding factor known as CTCF. The centromeric (domain 2) differentially methylated region (KvDMR) is located in exon 10 of KCNQ1OT1, along with the promotor region of this gene that is expressed in a tissue-specific manner normally only from the paternal allele. The KCNQ1OT1 transcript apparently cis-regulates the expression of other genes in domain 2. Loss of methylation (LOM) in $K C N Q 10 T 1$ results in abnormal expression of the maternal allele, observed in 50-60\% of BWS cases (Smith et al., 2007; Nativio et al., 2011; Romanelli et al., 2011).

Previous studies on the methylation status of DMRH19 and KvDMR in WT patients were based on time-consuming protocols (von Kanel et al., 2010) such as restriction fragment length polymorphism (RFLP) (Brown et al., 2008), combined bisulfite restriction analysis (COBRA) (Satoh et al., 2006; Brown et al., 2008; Cerrato et al., 2008), blotting analysis with a methylation-sensitive restriction enzyme assay (Schneid et al., 1993; Moulton et al., 1994; Taniguchi et al., 1995; Squire et al., 2000), real-time PCR coupled with a methylation-sensitive restriction assay (Bruce et al., 2008; Gomes et al., 2009) and reverse transcriptase-PCR coupled to a methylation-sensitive restriction enzyme (Okamoto et al., 1997; Squire et al., 2000).

In this work, we used a novel, rapid method of pyrosequencing, a technique that enables high resolution quantitative analysis of DNA methylation, and methylation sensitive-multiplex ligation-dependent probe amplification (MS-MLPA), a technique that allows the identification of changes in methylation patterns, to compare the constitu- tional and somatic methylation patterns of DMRH19 and KvDMR in 40 WT patients.

\section{Material and Methods}

\section{Subjects}

Forty patients (24 males, 16 females) with documented WT, treated at the Instituto Nacional de Câncer (Rio de Janeiro, Brazil), were included in this study. Peripheral blood DNA samples from $31 / 40$ patients and 34 fresh tumor DNA samples (from 29/40 patients) were used. All tumor samples were derived from primary tumors. For five patients more than one fresh tumor sample per patient (obtained at different times) was included: in two cases the first tumor sample was obtained from a biopsy while in three patients the samples were obtained from partial nephrectomies. All of the patients received with the same neoadjuvant chemotherapy treatment based on the International Society of Paediatric Oncology (SIOP) WT 2001 trial protocol (Bhatnagar, 2009). The patients were examined by a medical geneticist trained in dysmorphology. This study was approved by the Instituto Nacional de Câncer Ethics Committee (protocol number 87/08). All of the patients provided informed consent that was signed by parents or tutors.

\section{Methods}

DNA was extracted from peripheral blood and fresh tumor samples, essentially as described by Miller et al. (1988) and Sambrook et al. (1989). Pyrosequencing reactions were done with $2 \mu \mathrm{g}$ of DNA previously modified with bisulfite (EZ DNA Methylation kit, Zymo Research, CA, USA) according to the manufacturer's instructions. Primers complementary to the bisulfite-modified $\mathrm{CpG}$ islands in DMRH19 and KvDMR domains were designed with PSQ Assay Design Software v. 1.0.6 (Pyrosequencing AB, Uppsala, Sweden). Fragments spanning both domains were amplified with the following primers: DMRH19-F: GGGGTTATTTGGGAATAGGATAT; DMRH19-R: TAACTTAAATCCCAAACCATAACA; KvDMR-F: TGTTTAGGTTAGGTTGTATTGTTG; KvDMR-R: CCCATCTCTCTAAAAAAATTT. Reverse primers were biotinylated. The PCR mixtures contained $30 \mathrm{ng}$ of modified DNA, 7.5 pmol of forward and reverse primers (Roche Applied Science ${ }^{\circledR}$, Spain), $7.5 \mathrm{mM}$ dNTPs, $3 \mu \mathrm{L}$ of PCR buffer (Roche Applied Science ${ }^{\circledR}$ ) and $0.5 \mathrm{U}$ of Taq DNA polymerase (Roche Applied Science ${ }^{\circledR}$ ) in a final volume of $30 \mu \mathrm{L}$. The PCR conditions for the DMRH19 assay consisted of $95^{\circ} \mathrm{C}$ for $5 \mathrm{~min}, 45$ cycles at $95{ }^{\circ} \mathrm{C}$ for $30 \mathrm{~s}, 60^{\circ} \mathrm{C}$ for $30 \mathrm{~s}, 72^{\circ} \mathrm{C}$ for $30 \mathrm{~s}$ and $72{ }^{\circ} \mathrm{C}$ for $10 \mathrm{~min}$. The corresponding conditions for the KvDMR assay were $95^{\circ} \mathrm{C}$ for $5 \mathrm{~min}, 45$ cycles at $95^{\circ} \mathrm{C}$ for $30 \mathrm{~s}, 54^{\circ} \mathrm{C}$ for $30 \mathrm{~s}, 72^{\circ} \mathrm{C}$ for $30 \mathrm{~s}$ and $72^{\circ} \mathrm{C}$ for $10 \mathrm{~min}$. Single-stranded DNA templates 
and pyrosequencing were prepared and run according to the Biotage $\mathrm{AB}^{\circledR}$ protocol (Uppsala, Sweden) in a PSQHS96A system (Pyrosequencing $\mathrm{AB}^{\circledR}$, Uppsala, Sweden). Pyrosequencing was done with sequencing primers for each PCR product: GAATAGGATATTTATGGGAG for DMRH19-SF and GGGTATATAGTTTATTTTAGTA for KvDMR-SF (Roche Applied Science ${ }^{\circledR}$ ). The CpG peaks were analyzed and the final methylation index (MI) was calculated from the average of the percentage for each $\mathrm{CpG}$ peak in the pyrogram. The data were analyzed with Pyro Q-CpG software v. 1.0.9 (Pyrosequencing AB). Standard deviations (SD) were calculated based on the average MI and the distribution of MI values for the patients and healthy controls.

The MS-MLPA 11 p15 region kit SALSA ME030-B2 BWS/SRS (MRC-Holland, Amsterdam, Holland) contains 45 probes, 27 of which are specific for the BWS/SRS (Silver Russel Syndrome) region in $11 \mathrm{p} 15$. This kit was used with $300 \mathrm{ng}$ of genomic DNA and the methylationsensitive restriction enzyme HhaI (Promega Corporation, Spain) according to the manufacturer's protocol. The MSMLPA PCR products were analyzed in an ABI 3130 automated platform (Applied Biosystems ${ }^{\circledR}$, Foster City, CA, USA). The raw data were analyzed with Excel-based in- house software (Meth-HULP v. 1.1) developed by INGEMM (Instituto de Genética Médica y Molecular, Hospital Universitario La Paz, Madrid, Spain) (Romanelli et al., 2011). Normalization of the peak areas of undigested and digested samples from patients and controls (blood and kidney DNA samples) allowed visualization of the MI for each locus and sample. Methylation analysis was done by comparing restriction digested aliquots with paired undigested aliquots. Standard deviations were calculated based on the average MI and the distribution of MI values for the patients and healthy controls for each region (DMRH19 and KvDMR) analyzed.

\section{Results}

In this study, we investigated the constitutional and somatic 11 p15 methylation patterns in 24 males and 16 females with WT. There were four cases of bilateral tumor. The age at diagnosis ranged from five months to 137 months, with an average age at diagnosis of 43 months for all 40 patients and 44 months for patients with unilateral tumor. Diffuse or focal anaplasia was observed in five tumors (Table 1) and four of them also presented DMRH19 hypermethylation (Table 2). Perilobar nephrogenic rests (PLNR)

Table 1 - Clinical and histopathological data for 40 patients with Wilms tumor.

\begin{tabular}{|c|c|c|c|c|c|}
\hline Subject & Gender & Laterality & Age $d x$ & Histopathology & Phenotype \\
\hline P1 & $\mathrm{F}$ & $\mathrm{U}$ & 25 & Tri & WAGR \\
\hline P2 & M & $\mathrm{B}$ & 13 & Tri (+) B1. & BWS \\
\hline P4 & M & $\mathrm{U}$ & 5 & Tri ILNR & \\
\hline P5 & $\mathrm{F}$ & $\mathrm{B}$ & 9 & Tri (+) Bl. & \\
\hline P6 & M & $\mathrm{U}$ & 30 & Tri & uds \\
\hline P7 & $\mathrm{F}$ & $\mathrm{U}$ & 45 & Tri & $\mathrm{HH}$ \\
\hline P9 & $\mathrm{F}$ & $\mathrm{U}$ & 47 & Tri & \\
\hline $\mathrm{P} 10$ & $\mathrm{~F}$ & $\mathrm{U}$ & 10 & Tri & \\
\hline P11 & M & $\mathrm{U}$ & 136 & $\mathrm{~B} 1$ & \\
\hline $\mathrm{P} 12$ & M & $\mathrm{U}$ & 90 & $\mathrm{~B} 1$ & \\
\hline $\mathrm{P} 13$ & $\mathrm{~F}$ & $\mathrm{U}$ & 62 & Ep & \\
\hline P14 & M & $\mathrm{U}$ & 59 & Ep & \\
\hline P15 & $\mathrm{F}$ & $\mathrm{U}$ & 62 & Tri & \\
\hline P16 & M & $\mathrm{U}$ & 28 & DA & \\
\hline \multirow[t]{2}{*}{ P17 } & M & $\mathrm{B}$ & 50 & Ep (FTS A) & \\
\hline & & & & FA (FTS B) & \\
\hline P18 & M & $\mathrm{U}$ & 36 & FA & \\
\hline P19 & $\mathrm{F}$ & $\mathrm{U}$ & 48 & Tri (FTS A and B) & \\
\hline P20 & M & $\mathrm{U}$ & 137 & Tri & \\
\hline \multirow[t]{2}{*}{ P21 } & $\mathrm{F}$ & $\mathrm{U}$ & 5 & B1 (FTS A) & \\
\hline & & & & Tri (FTS B) & \\
\hline P22 & $\mathrm{F}$ & $\mathrm{U}$ & 29 & Tri (FTS B and C) & macr \\
\hline P23 & M & $\mathrm{U}$ & 57 & FA & \\
\hline
\end{tabular}


Table 1 (cont.)

\begin{tabular}{|c|c|c|c|c|c|}
\hline Subject & Gender & Laterality & Age $d x$ & Histopathology & Phenotype \\
\hline P24 & $\mathrm{F}$ & $\mathrm{U}$ & 13 & Tri & \\
\hline P25 & M & $\mathrm{U}$ & 42 & Tri & \\
\hline P26 & M & $\mathrm{U}$ & 57 & Tri & HH Moe \\
\hline \multirow[t]{2}{*}{ P27 } & M & $\mathrm{B}$ & 61 & Ep PLNR (FTS B) & \\
\hline & & & & Tri PLNR (FTS C) & \\
\hline P28 & M & $\mathrm{U}$ & 67 & Tri & \\
\hline P29 & $\mathrm{F}$ & $\mathrm{U}$ & 49 & Ep & \\
\hline P31 & $\mathrm{F}$ & $\mathrm{U}$ & 34 & Tri & \\
\hline P32 & $\mathrm{F}$ & $\mathrm{U}$ & 6 & Bl & \\
\hline P33 & M & $\mathrm{U}$ & 54 & B1 & \\
\hline P34 & $\mathrm{F}$ & $\mathrm{U}$ & 35 & Tri & \\
\hline P35 & M & $\mathrm{U}$ & 46 & Tri & BWS \\
\hline P36 & M & $\mathrm{U}$ & 32 & $\mathrm{B1} ; \mathrm{DA}$ & \\
\hline P37 & M & $\mathrm{U}$ & 48 & Tri & \\
\hline P38 & $\mathrm{F}$ & $\mathrm{U}$ & 19 & Bl/Ep PLNR & macr \\
\hline P39 & M & $\mathrm{U}$ & 48 & Tri & \\
\hline P40 & M & $\mathrm{U}$ & 28 & Tri & \\
\hline P41 & M & $\mathrm{U}$ & 26 & $\mathrm{~B} 1$ & \\
\hline P42 & M & $\mathrm{U}$ & 56 & $\mathrm{St}$ & \\
\hline P43 & M & $\mathrm{U}$ & 13 & Tri & \\
\hline Total $(\mathrm{P}): 40$ & & & $43 *$ & & \\
\hline
\end{tabular}

(A), (B) and (C) - tumor samples A, B and C, respectively. Age dx - age at diagnosis (months), B - bilateral, Bl - blastemal, Ep - epithelial, DA - diffuse anaplasia, F - female, FA - focal anaplasia, FTS - fresh tumor sample, HH - hemihypertrophy, ILNR - intralobar nephrogenic rests, M - male, macr macrosomia, P - patient, PLNR - perilobar nephrogenic rests, Tri - triphasic, St - stromal, U - unilateral, uds - undiagnosed dysmorphic syndrome. *Average age at diagnosis.

Table 2 - Pyrosequencing and MS-MLPA for 11p15.

\begin{tabular}{|c|c|c|c|c|}
\hline \multirow[t]{3}{*}{ Subject } & \multicolumn{4}{|c|}{ DMRH19/KvDMR pyrosequencing and MS-MLPA } \\
\hline & \multicolumn{2}{|c|}{ Peripheral blood DNA samples } & \multicolumn{2}{|c|}{ Fresh tumor DNA samples } \\
\hline & Pyrosequencing & MS-MLPA & Pyrosequencing & MS-MLPA \\
\hline P1 & $\mathrm{N}$ & $\mathrm{N}$ & NA & NA \\
\hline P2 & HM DMRH19 & HM DMRH19 & NA & NA \\
\hline P4 & $\mathrm{N}$ & $\mathrm{N}$ & NA & NA \\
\hline P5 & $\mathrm{N}$ & $\mathrm{N}$ & NA & NA \\
\hline P6 & $\mathrm{N}$ & $\mathrm{N}$ & NA & NA \\
\hline P7 & $\mathrm{N}$ & $\mathrm{N}$ & NA & NA \\
\hline P9 & $\mathrm{N}$ & $\mathrm{N}$ & NA & NA \\
\hline P10 & $\mathrm{N}$ & $\mathrm{N}$ & NA & NA \\
\hline P11 & $\mathrm{N}$ & $\mathrm{N}$ & HM DMRH19 & HM DMRH19 \\
\hline $\mathrm{P} 12$ & $\mathrm{~N}$ & $\mathrm{~N}$ & HM DMRH19 & HM DMRH19 \\
\hline P13 & NA & $\mathrm{N}$ & UPD & UPD \\
\hline P14 & $\mathrm{N}$ & $\mathrm{N}$ & $\mathrm{N}$ & $\mathrm{N}$ \\
\hline P15 & $\mathrm{N}$ & $\mathrm{N}$ & $\mathrm{N}$ & LOM DMRH19 \\
\hline P16 & $\mathrm{N}$ & $\mathrm{N}$ & HM DMRH19 & HM DMRH19 \\
\hline \multirow[t]{2}{*}{ P17 } & $\mathrm{N}$ & $\mathrm{N}$ & HM DMRH19 (A) & HM DMRH19 (A) \\
\hline & & & HM DMRH19 (B) & HM DMRH19 (B) \\
\hline
\end{tabular}


Table 2 (cont.)

\begin{tabular}{|c|c|c|c|c|}
\hline \multirow[t]{3}{*}{ Subject } & \multicolumn{4}{|c|}{ DMRH19/KvDMR pyrosequencing and MS-MLPA } \\
\hline & \multicolumn{2}{|c|}{ Peripheral blood DNA samples } & \multicolumn{2}{|c|}{ Fresh tumor DNA samples } \\
\hline & Pyrosequencing & MS-MLPA & Pyrosequencing & MS-MLPA \\
\hline $\mathrm{P} 18$ & $\mathrm{~N}$ & $\mathrm{~N}$ & HM DMRH19 & HM DMRH19 \\
\hline \multirow[t]{2}{*}{ P19 } & $\mathrm{N}$ & $\mathrm{N}$ & UPD (A) & $\mathrm{UPD}(\mathrm{A})$ \\
\hline & & & UPD (B) & UPD (B) \\
\hline $\mathrm{P} 20$ & $\mathrm{~N}$ & $\mathrm{~N}$ & $\mathrm{~N}$ & $\mathrm{~N}$ \\
\hline \multirow[t]{2}{*}{$\mathrm{P} 21$} & NA & NA & UPD (FTS A) DMRH19 MI = 79\%* & UPD (A) \\
\hline & & & $\mathrm{N}($ FTS B) DMRH19 MI = 42\%* & $\mathrm{N}(\mathrm{B})$ \\
\hline \multirow[t]{2}{*}{$\mathrm{P} 22$} & $\mathrm{~N}$ & $\mathrm{~N}$ & HM DMRH19 (B) & HM DMRH19 (B) \\
\hline & & & HM DMRH19 (C) & HM DMRH19 (C) \\
\hline $\mathrm{P} 23$ & NA & NA & HM DMRH19 & HM DMRH19 \\
\hline P24 & NA & NA & UPD & UPD \\
\hline P25 & $\mathrm{N}$ & $\mathrm{N}$ & HM DMRH19 & HM DMRH19 \\
\hline P26 & $\mathrm{N}$ & $\mathrm{N}$ & NA & NA \\
\hline \multirow[t]{2}{*}{ P27 } & $\mathrm{N}$ & $\mathrm{N}$ & HM DMRH19 (B) & HM DMRH19 (B) \\
\hline & & & HM DMRH19 (C) & HM DMRH19 (C) \\
\hline P28 & $\mathrm{N}$ & $\mathrm{N}$ & UPD & UPD \\
\hline P29 & NA & NA & UPD & UPD \\
\hline P31 & $\mathrm{N}$ & $\mathrm{N}$ & UPD & UPD \\
\hline P32 & NA & NA & UPD & UPD \\
\hline P33 & $\mathrm{N}$ & $\mathrm{N}$ & NA & NA \\
\hline P34 & $\mathrm{N}$ & $\mathrm{N}$ & HM DMRH19 & HM DMRH19 \\
\hline P35 & HM DMRH19 & NA & UPD & UPD \\
\hline P36 & NA & NA & $\mathrm{N}$ & $\mathrm{N}$ \\
\hline P37 & NA & NA & HM DMRH19 & HM DMRH19 \\
\hline P38 & $\mathrm{N}$ & $\mathrm{N}$ & HM DMRH19 & HM DMRH19 \\
\hline P39 & $\mathrm{N}$ & $\mathrm{N}$ & NA & NA \\
\hline P40 & $\mathrm{N}$ & $\mathrm{N}$ & UPD & UPD \\
\hline P41 & $\mathrm{N}$ & $\mathrm{N}$ & $\mathrm{N}$ & $\mathrm{N}$ \\
\hline P42 & $\mathrm{N}$ & $\mathrm{N}$ & UPD & UPD \\
\hline P43 & NA & NA & HM DMRH19 & HM DMRH19 \\
\hline Total (P): 40 & $31 / 40$ & $31 / 40$ & $34 / 40$ & $34 / 40$ \\
\hline
\end{tabular}

(A), (B) and (C), tumor samples A, B and C, respectively. FTS - fresh tumor sample, HM - hypermethylation, LOM - loss of methylation, N - normal, NA - not available, P - patient, UPD - uniparental disomy. *P21 MI: DMRH19 methylation index for tumor samples from patient 21.

were observed in two patients and intralobar rests (ILNR) in one patient. One patient with PLNR (P38) had macrosomia since birth and mild indentations on the ear lobes, but no other findings compatible with the clinical features of BWS. Eight patients had phenotypic abnormalities: one had WAGR syndrome (P1), two had BWS (P2 and P35), two had macrosomia defined by stature and weight above the $97^{\text {th }}$ centile for age and sex (P22 and P38), two had hemihypertrophy (P7 and P26), one of whom (P26) also presented with Moebius sequence (bilateral VII cranial nerve paralysis), and the eighth patient (P6) had an undiagnosed dysmorphic syndrome (Table 1).
Pyrosequencing of DMRH19 in 31 blood DNA samples detected hypermethylation in the two BWS patients (Table 2), with MI of $71 \%$ and $57 \%$, respectively; these values were higher than in healthy blood controls ( $\mathrm{MI}=45.4 \pm$ $6.2 \%$ ) (Figure 1). Pyrosequencing of KvDMR in 31 blood samples yielded normal MI estimates (Table 2, Figure 2), with the corresponding MI for healthy blood (control) being $38.9 \pm 3.1 \%$.

Pyrosequencing of DMRH19 in 34 fresh tumor samples detected hypermethylation in 28 samples (Table 2). In five patients with more than one tumor sample, analysis of the two samples obtained at different times yielded similar 
A

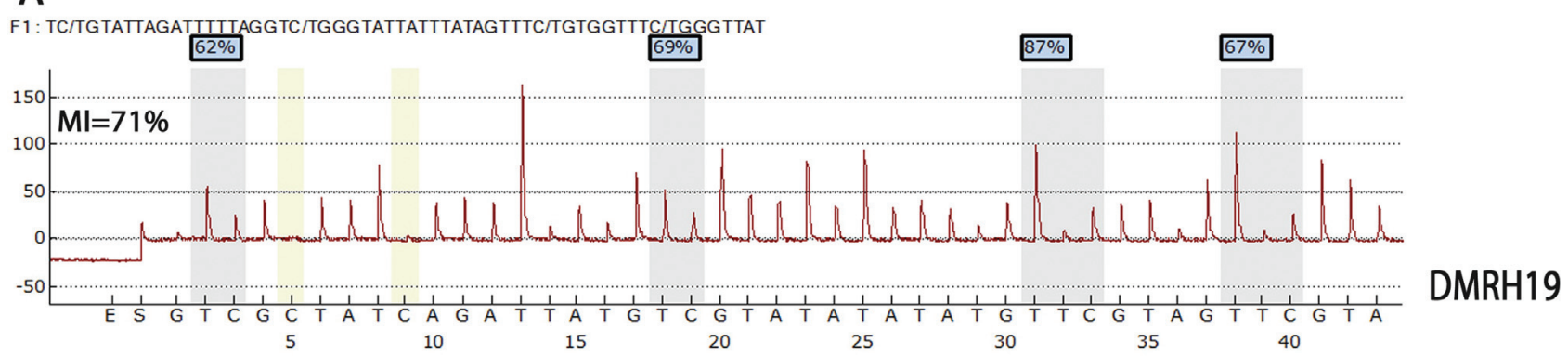

B

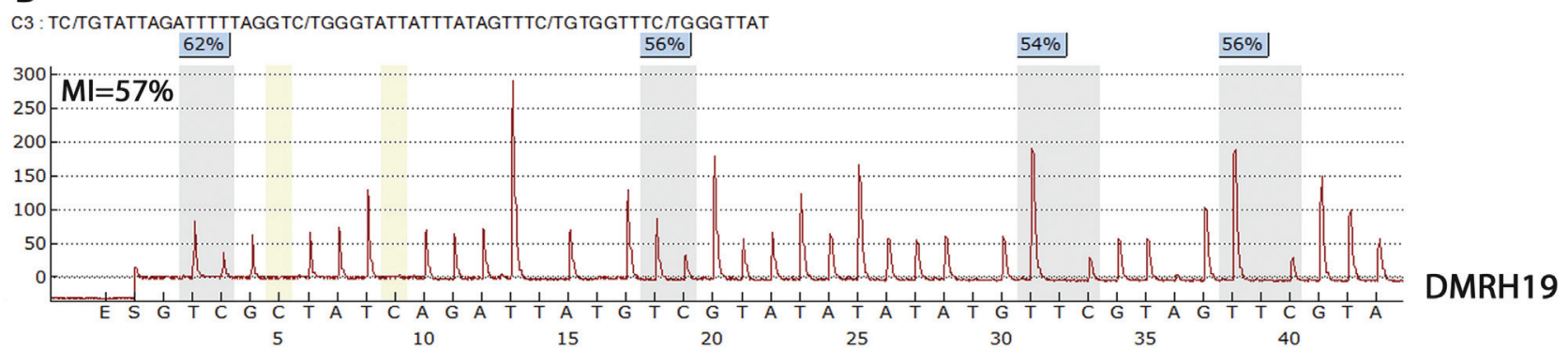

C

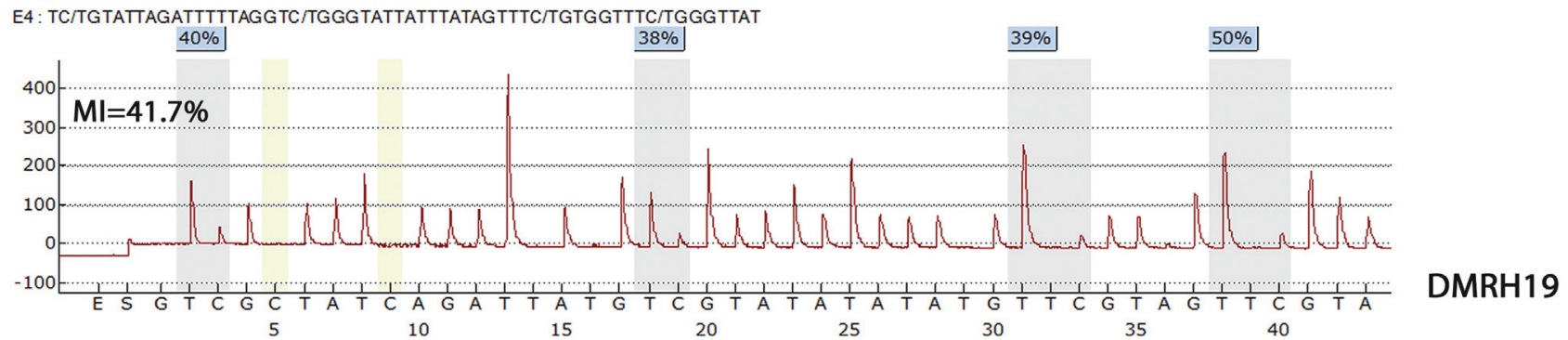

Figure 1 - Pyrogram showing DMRH19 hypermethylation in blood DNA samples from patients P2 (A) and P35 (B). The average methylation index (MI) for DMRH1 9 in patients $\mathrm{P} 2$ and P35 was 71\% and 57\%, respectively. Panel (C) shows DMRH19 methylation in blood from healthy controls (average MI $=41.7 \%)$. The average MI of all blood samples from healthy controls used in DMRH19 pyrosequencing was $45.4 \pm 6.2 \%$. The MI is indicated above peaks (gray columns) corresponding to the $\mathrm{CpG}$ islands in this region.

estimates of MI, except for one patient (p21) with an MI of $79 \%$ in sample A and $42 \%$ in sample B (Table 2, Figure 3 ). The corresponding MI for healthy renal tissue (control) was $42.6 \pm 15.3 \%$.

KvDMR pyrosequencing in 34 fresh tumor DNA samples detected LOM in 12 samples (Table 2). The MI in healthy renal tissue (control) was $39.2 \pm 10.8 \%$. Pyrosequencing showed that these 12 tumor samples with KvDMR LOM also had DMRH19 hypermethylation that was characteristic of paternal uniparental disomy (UPD) (Table 2).

MS-MLPA was done in 31 peripheral blood samples and in all 34 tumor samples (Table 2). DMRH19 hypermethylation was observed in only one peripheral blood sample (P2); the remaining blood samples were normal (Figure 4A,B). MS-MLPA analysis of tumor samples showed isolated DMRH19 hypermethylation in 16/34 cases and in 12/34 samples a characteristic pattern of paternal UPD was observed (Table 2, Figure 4C). Isolated
DMRH19 LOM was detected in one tumor sample (P15) while a normal methylation pattern was observed in the remaining 5/34 samples (Table 2).

\section{Discussion}

In this study, we used pyrosequencing and MSMLPA to analyze the constitutional and somatic methylation patterns in the DMRH19 and KvDMR regions of 40 WT patients. Data from tumor histopathology and physical examination were compared with the methylation status in $11 \mathrm{p} 15$. The comparison of peripheral blood and fresh tumor samples confirmed that the epigenetic changes associated with WT were mostly somatic. Phenotypic abnormalities characteristic of a syndromic form of WT were observed in $20 \%$ of patients $(8 / 40)$. The average age at diagnosis in our cohort was 43 months, which was similar to the 38 months reported by Scott et al. (2012). 
A

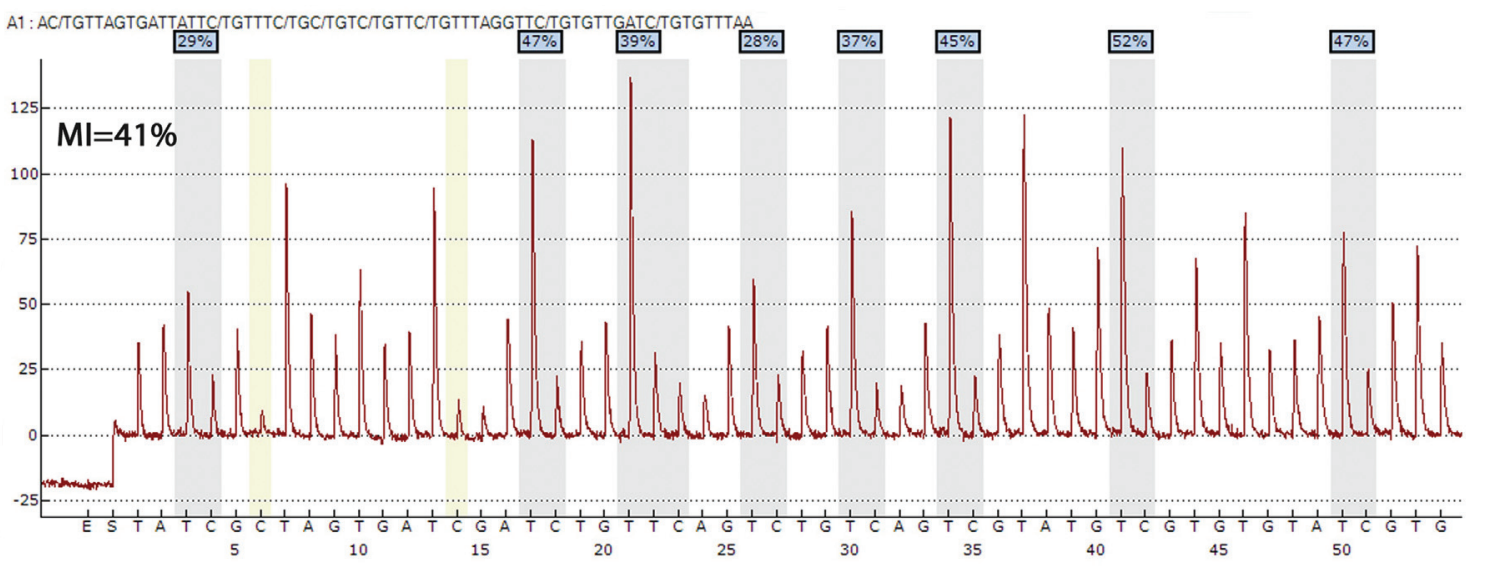

KvDMR

B

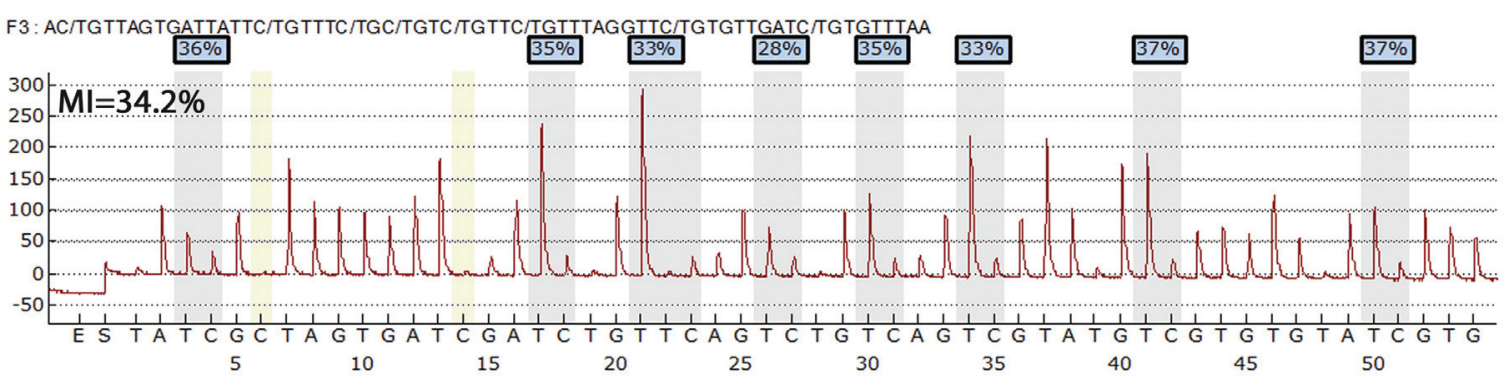

KvDMR

C

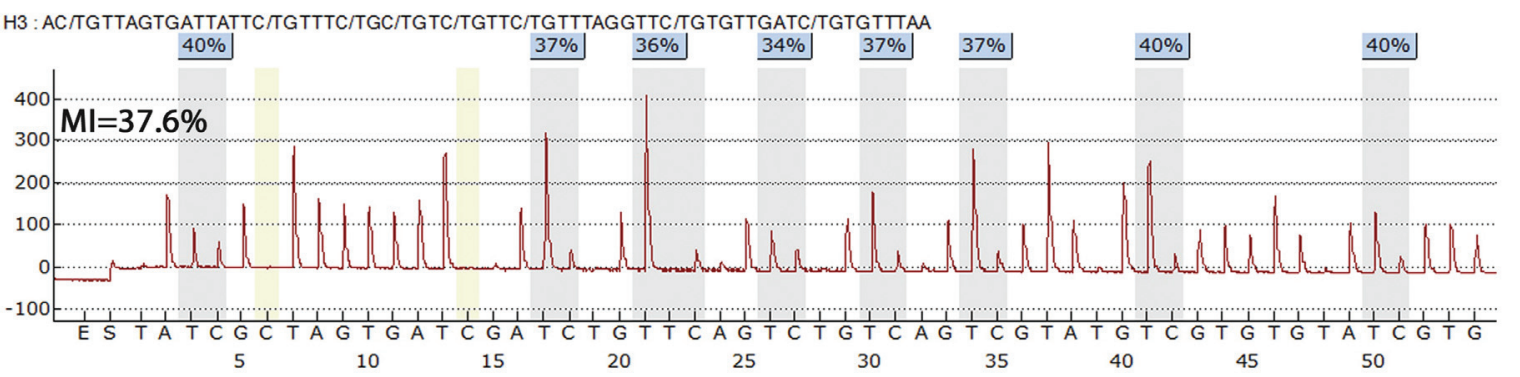

KvDMR

Figure 2 - Pyrogram showing normal methylation of KvDMR. (A) P2 blood sample (average MI = 41\%), (B) P35 blood sample (average MI = 34.2\%) and $(\mathrm{C})$ healthy control blood sample (average $\mathrm{MI}=37.6 \%$ ). The average MI of all blood samples from healthy controls used in KvDMR pyrosequencing was $38.9 \pm 3.1 \%$. The $\mathrm{MI}$ is indicated above peaks (gray columns) corresponding to the $\mathrm{CpG}$ islands in this region.

Four patients had bilateral WT and tumor samples were available from two of them (P17 and P27). Two tumor samples from each of these patients were studied, one from each kidney (left and right). Sample A from patient P17 had a predominantly epithelial histology while sample $\mathrm{B}$, which was collected from a subsequent nephrectomy on the contralateral kidney, showed focal anaplasia that probably reflected evolution of the WT. Both samples had DMRH19 hypermethylation and the DNA methylation indices were similar in the two samples from each of these patients.

Constitutional DMRH19 hypermethylation was observed in only two patients (P2 and P35), both with the BWS phenotype. These patients fit into a small group (5-7\%) of BWS patients with isolated DMRH19 hypermethylation reported by Bliek et al. (2001). Interestingly, these authors found that two of the four BWS patients who showed isolated DMRH19 hypermethylation also developed WT (Bliek et al., 2001). The normal constitutional methylation profiles shown by KvDMR pyrosequencing in the present study confirmed that most epigenetic alterations associated with WT involve DMRH19 but not KvDMR, as also reported by Priolo et al. (2008).

Eight of our patients also had major phenotypic abnormalities, including two patients with BWS (P2 and P35), two patients with hemihypertrophy (P7 and P26), two with isolated macrosomia (P22 and P38), one patient with WAGR complex (P1) and one patient with an undiagnosed dysmorphic syndrome (P6). Except for Moebius syndrome, the occurrence of which in patient P26 may have been fortuitous, all of the other pathogenic conditions have been re- 
A

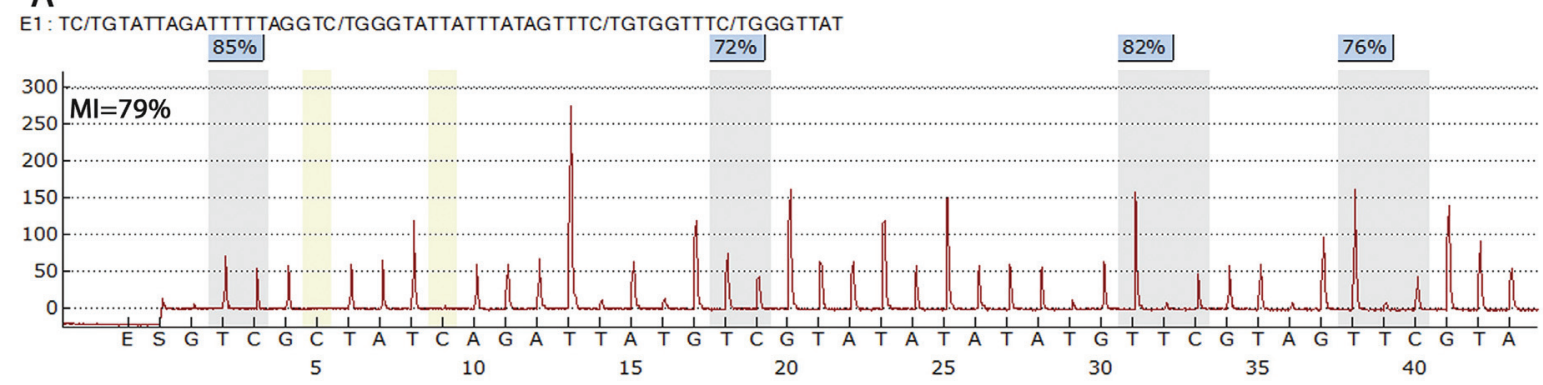

DMRH19

B

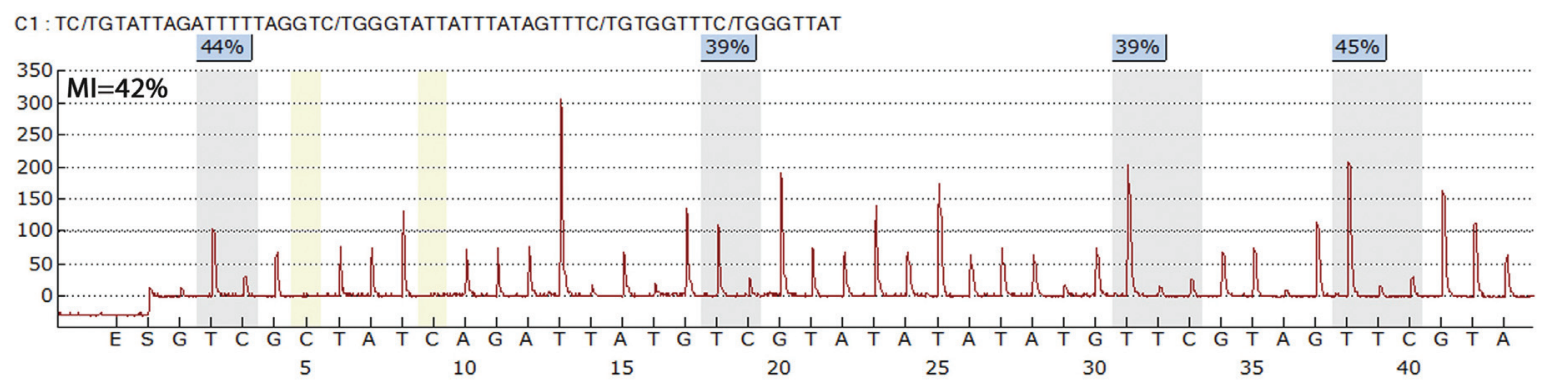

DMRH19

C

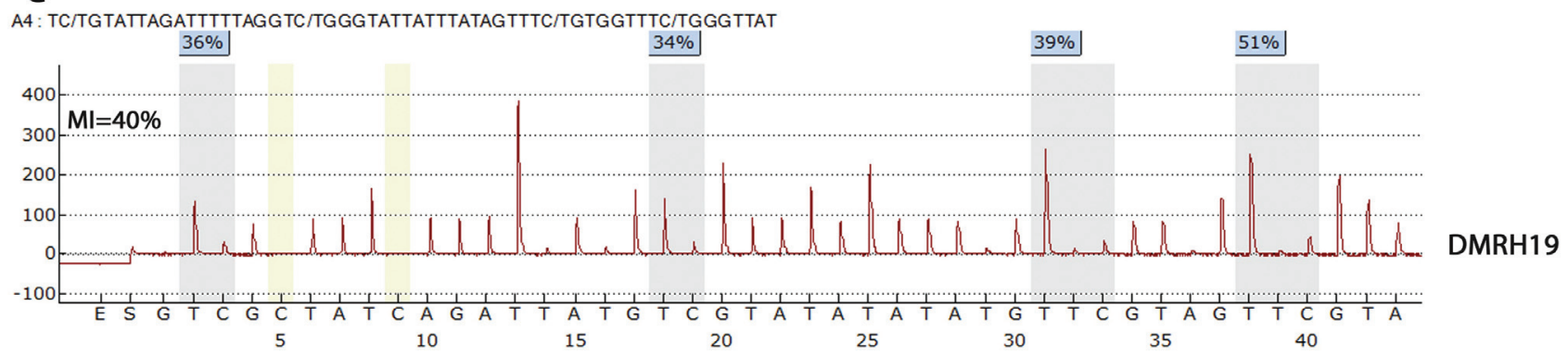

Figure 3 - Pyrogram showing DMRH19 hypermethylation in renal tumor samples from patient P21. (A) Tumor sample A (average MI $=79 \%$ ). (B) Tumor sample $\mathrm{B}$ (average $\mathrm{MI}=42 \%$ ). (C) Control sample from healthy kidney (average $\mathrm{MI}=40 \%$ ). The average of all healthy control renal samples used in DMRH19 pyrosequencing was $42.6 \pm 15.3 \%$. The MI is indicated above peaks (gray columns) corresponding to the CpG islands in this region.

currently described in association with WT, indicating the heterogeneous etiology of this neoplasia (Dome and Huff, 2011). As already described in a proportion of these patients, constitutive DMRH19 hypermethylation was observed in both BWS patients and somatic UPD was present in patient P35. Perilobar nephrogenic rests were observed in one of the patients with macrosomia (P38), as described above, and her tumor sample showed DMRH19 hypermethylation.

DMRH19 pyrosequencing showed isolated hypermethylation in $47 \%(16 / 34)$ of tumor samples, which was lower than the $71 \%$ previously reported with the COBRA assay in 28 WT samples (Brown et al., 2008). However, another study based on COBRA analysis (Cerrato et al., 2008) detected isolated somatic DMRH19 hypermethylation in $10 / 40$ WT patients (25\%). Contrary to our study, in which 12 patients showed somatic LOM of KvDMR, Brown et al. (2008) observed no differences in the extent of KvDMR methylation between normal and tumor samples. Scott et al. (2012) reported that the proportion of isolated
DMRH19 hypermethylation observed with MS-MLPA was $31 \%$, a value similar to our results.

One of our patients (P21) showed a difference in the extent of methylation between tumor samples A (79\%) and B (42\%). This discrepancy may reflect the fact that sample B was collected after successful chemotherapy with $90 \%$ tumor regression. DNA methylation levels are known to be influenced by various chemotherapeutic regimens. Among our patients, with exception of two biopsy samples, the remaining tumor samples were obtained from patients who had been submitted to the same chemotherapeutic SIOP protocol (Bhatnagar, 2009). Variations in the rate of tumor regression in response to chemotherapy may have an impact on DNA methylation levels, as observed in patient P21.

Isolated DMRH19 hypermethylation and paternal UPD result in $I G F 2$ biallelic expression and, subsequently, tumor development (Beckwith, 1998). The pyrosequencing of KvDMR in tumor samples revealed LOM in 12/34 samples, concomitant with DMRH19 hypermethylation, indi- 


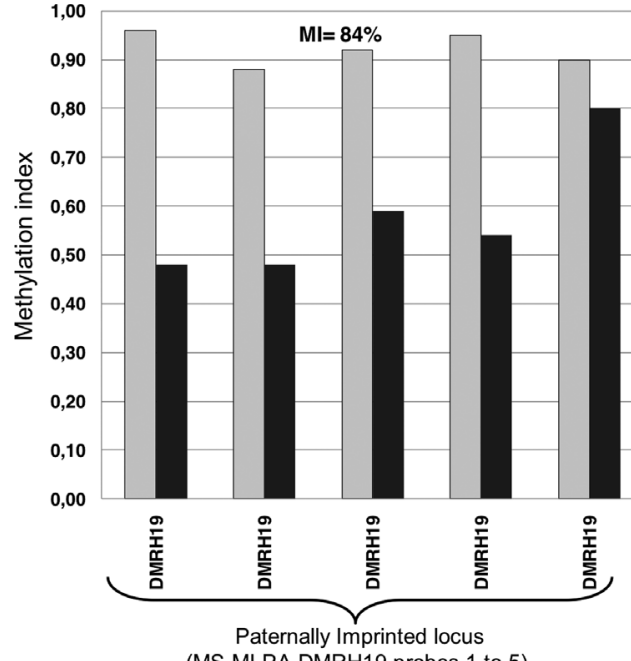

(MS-MLPA DMRH19 probes 1 to 5 )
$M I=64 \%$
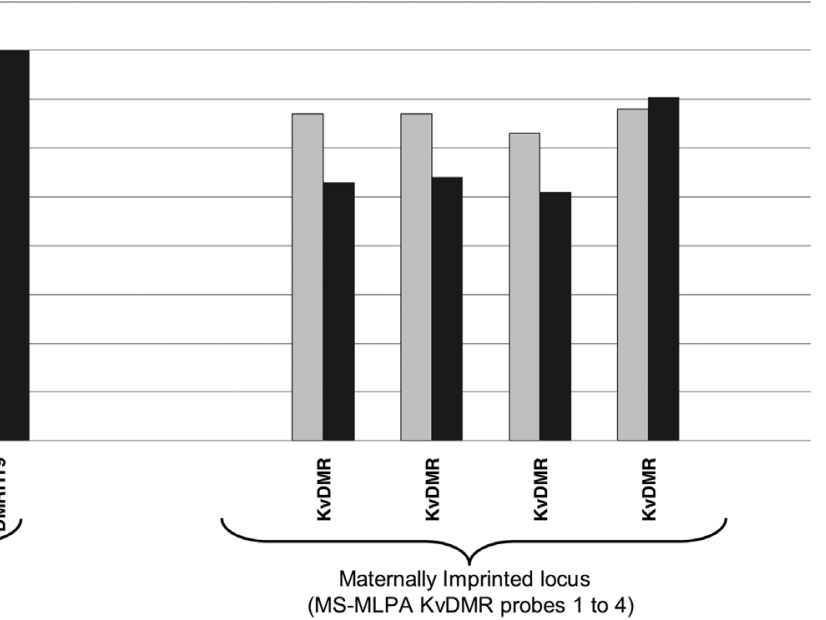

(MS-MLPA KvDMR probes 1 to 4 )

A

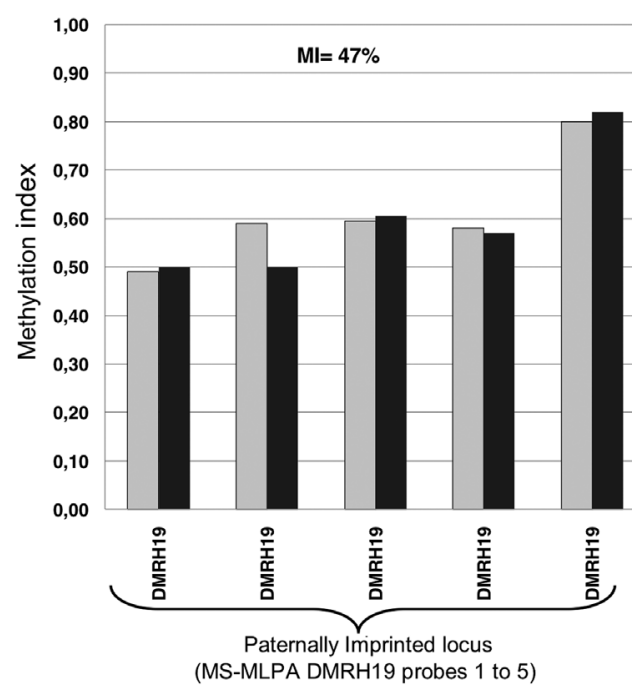

$\mathrm{Ml}=\mathbf{5 1} \%$

B

sample
Control (mean)
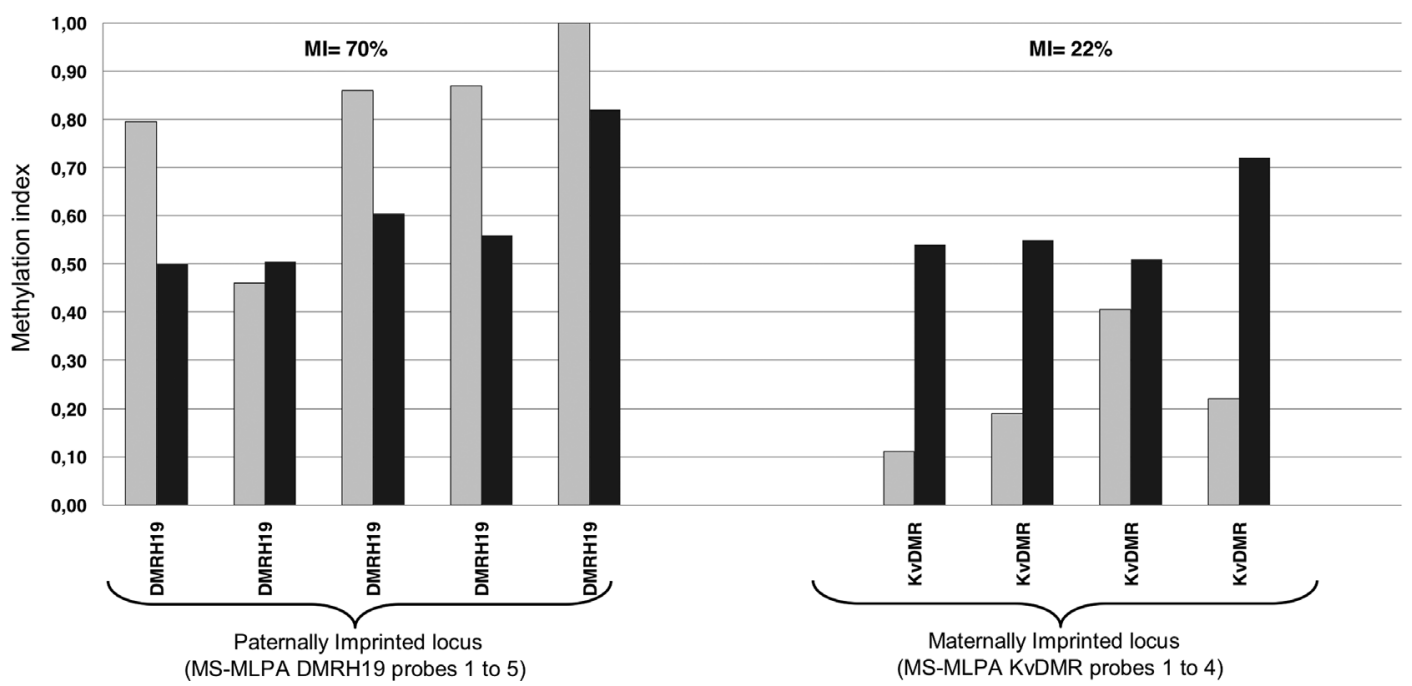

C

P19 tumor DNA

sample A

Control (mean)

Figure 4 - MS-MLPA methylation indices. (A) Peripheral blood DNA from patient P2 showing isolated hypermethylation of DMRH19 (average MI = 84 $\pm 6 \%$ ) and normal methylation of KvDMR (average MI $=64 \pm 8 \%$ ). (B) Peripheral blood DNA from patient P42 showing normal methylation of DMRH19 (average MI $=47 \pm 6 \%$ ) and KvDMR (average MI $=51 \pm 8 \%$ ). (C) Fresh tumor DNA (sample A) from patient P19 showing paternal uniparental disomy (average MI for DMRH19 and KvDMR was $70 \pm 27 \%$ and $22 \pm 19 \%$, respectively). Dark gray columns: expected ratios; light gray columns: observed ratios. Each pair of columns corresponds to one of the DMRH19 and KvDMR probes included in the MS-MLPA kit (five probes for DMRH19 and four for KvDMR). 
cating paternal UPD in 11 out of 29 patients (38\%). This proportion was similar to a previous UPD estimate (45\%) in 40 WT patients (Cerrato et al., 2008).

We used MS-MLPA as a complementary procedure to analyze DMRH19 and KvDMR methylation. This assay yielded findings consistent with those obtained by pyrosequencing, except for one tumor sample (P15) that showed DMRH19 LOM, probably because of a technical artefact. In addition, MS-MLPA confirmed the somatic, paternal UPD in 12 tumor samples.

Few studies have compared constitutional and somatic $11 \mathrm{p} 15$ epigenetic alterations in WT patients. One of these used MS-MLPA to analyze blood DNA samples from 437 children with non-syndromic WT and identified constitutional defects in 13 patients (3\%), including six patients with DMRH19 hypermethylation and six with paternal UPD (Scott et al., 2008b). Constitutional 11p15 defects were observed in 5\% (2/40) of our patients. The concomitant finding of macrosomia and somatic isolated DMRH19 hypermethylation in two patients (P22 and P38; Tables 1 and 2) indicated that these patients may represent somatic mosaics for this epigenetic disturbance. Additionally, patient P38 also had PLNR, a condition previously reported to be associated with this phenotype (Dome and Huff, 2011).

To the best of our knowledge, this is the first study to compare constitutional and somatic DMRH19 and KvDMR epigenetic alterations in WT patients by pyrosequencing and MS-MLPA. Our findings highlight the benefits of the combined analysis of the DMRH19 and KvDMR regions. The MS-MLPA and pyrosequencing findings were highly consistent. MS-MLPA is a rapid, reliable technique that is less expensive than pyrosequencing and is adequate for accurate quantitative estimation of DNA methylation in a clinical setting.

\section{Acknowledgments}

This work was supported by Conselho Nacional de Desenvolvimento Científico (CNPq; grants 401966/2010-0, 476808/2010-3, 573806/2008-0) and Fundação de Amparo à Pesquisa do Estado do Rio de Janeiro (FAPERJ; E26/170.026/2008). Leila C.A. Cardoso was supported by a CNPq visiting fellowship for her training at the Instituto de Genética Médica y Molecular, Hospital Universitario La Paz, Spain, as part of her studies for a $\mathrm{PhD}$ degree from the Post-Graduate Program in Genetics, Universidade Federal do Rio de Janeiro (UFRJ).

\section{References}

Beckwith JB (1998) Nephrogenic rests and the pathogenesis of Wilms tumor: Developmental and clinical considerations. Am J Med Genet 79:268-273.

Bhatnagar S (2009) Management of Wilms' tumor: NWTS vs. SIOP. J Indian Assoc Pediatr Surg 14:6-14.
Bliek J, Maas SM, Ruijter JM, Hennekam RCM, Alders M, Westerveld A and Mannens MMAM (2001) Increased tumour risk for BWS patients correlates with aberrant $H 19$ and not KCNQ1OT1 methylation: Occurrence of KCNQ1OT1 hypomethylation in familial cases of BWS. Hum Mol Genet 10:467-476.

Brown KW, Power F, Moore B, Charles AK and Malik KTA (2008) Frequency and timing of loss of imprinting at $11 \mathrm{p} 13$ and $11 \mathrm{p} 15$ in Wilms' tumour development. Mol Cancer Res 6:1114-1123.

Bruce S, Hannula-Jouppi K, Lindgren CM, Lipsanen-Nyman M and Kere J (2008) Restriction site-specific methylation studies of imprinted genes with quantitative real-time PCR. Clin Chem 54:491-499.

Cerrato F, Sparago A, Verde G, De Crescenzo, Citro V, Cubellis MV, Rinaldi MM, Boccuto L, Neri G, Magnani C, et al. (2008) Different mechanisms cause imprinting defects at the IGF2/H19 locus in Beckwith-Wiedemann syndrome and Wilm's tumour. Hum Mol Genet 17:1427-1435.

De Camargo B, de Oliveira Ferreira JM, de Souza Reis R, Ferman S, Santos MO and Pombo-de-Oliveira MS (2011) Socioeconomic status and the incidence of non-central nervous system childhood embryonic tumours in Brazil. BMC Cancer 11:160-166.

Gomes MV, Huber J, Ferriani RA, Amaral Neto AM and Ramos ES (2009) Abnormal methylation at the KvDMR 1 imprinting control region in clinically normal children conceived by assisted reproductive technologies. Mol Hum Reprod 15:471-477.

Miller RW, Young Jr JL and Novakovic B (1995) Childhood cancer. Cancer 75:395-405.

Miller SA, Dykes DD and Polesky HF (1988) A simple salting out procedure for extracting DNA from human nucleated cells. Nucleic Acids Res 16:1215.

Moulton T, Crenshaw T, Hao Y, Moosikasuwan J, Lin N, Dembitzer F, Hensle T, Weiss L, McMorrow L, Loew T, et al. (1994) Epigenetic lesions at the H19 locus in Wilms1 tumour patients. Nat Genet 7:440-447.

Nakamura L and Ritchey M (2010) Current management of Wilms' tumour. Curr Urol Rep 11:58-65.

Nativio R, Sparago A, Ito Y, Weksberg R, Riccio A and Murrell A (2011) Disruption of genomic neighbourhood at the imprinted IGF2-H19 locus in Beckwith-Wiedemann syndrome and Silver-Russell syndrome. Hum Mol Genet 20:13631374.

Okamoto K, Morison IM, Taniguchi T and Reeve AE (1997) Epigenetic changes at the insulin-like growth factor II/H19 locus in developing kidney is an early event in Wilms tumourigenesis. Proc Natl Acad Sci USA 94:5367-5371.

Priolo M, Sparago A, Mammì C, Cerrato F, Laganà C and Riccio A (2008) MS-MLPA is a specific and sensitive technique for detecting all chromosome $11 \mathrm{p} 15.5$ imprinting defects of BWS and SRS in a single-tube experiment. Eur J Hum Genet 16:565-571.

Romanelli V, Meneses HN, Fernández L, Martínez-Glez V, Gracia-Bouthelier R, Fraga FM, Guillén E, Nevado J, Gean E, Martorell L, et al. (2011) Beckwith-Wiedemann syndrome and uniparental disomy $11 \mathrm{p}$ : Fine mapping of the recombination breakpoints and evaluation of several techniques. Eur J Hum Genet 19:416-421. 
Sambrook J, Fritsch EF and Maniatis T (1989) Molecular Cloning: A Laboratory Manual. 2nd edition. Cold Spring Harbor Laboratory Press, New York, 545 pp.

Satoh Y, Nakadate H, Nakagawachi T, Higashimoto K, Joh K, Masaki Z, Uozumi J, Kaneko Y, Mukai T and Soejima H (2006) Genetic and epigenetic alterations on the short arm of chromosome 11 are involved in a majority of sporadic Wilm's tumours. Br J Cancer 95:541-547.

Schneid H, Seurin D, Vazquez MP, Gourmelen M, Cabrol S and Le Boue Y (1993) Parental allele specific methylation of the human insulin-like growth factor II gene and BeckwithWiedemann syndrome. J Med Genet 30:353-362.

Scott RH, Douglas J, Baskcomb L, Nygren AO, Birch JM, Cole TR, Cormier-Daire V, Eastwood DM, Garcia-Minaur S, Lupunzina P, et al. (2008a) Methylation-specific multiplex ligation-dependent probe amplification (MS-MLPA) robustly detects and distinguishes $11 \mathrm{p} 15$ abnormalities associated with overgrowth and growth retardation. J Med Genet 45:106-113.

Scott RH, Douglas J, Baskcomb L, Huxter N, Barker K, Hanks S, Craft A, Gerrard M, Kohler JA, Levitt GA, et al. (2008b) Constitutional $11 \mathrm{p} 15$ abnormalities, including heritable imprinting center mutations, cause nonsyndromic Wilms tumour. Nat Genet 40:1329-1334.

Scott RH, Murray A, Baskcomb L, Turnbull C, Loveday C, Al-Saadi R, Williams R, Breatnach F, Gerrard M, Hale J, et al. (2012) Stratification of Wilms tumor by genetic and epigenetic analysis. Oncotarget 3:327-335.
Smith AC, Choufani S, Ferreira JC and Weksberg R (2007) Growth regulation, imprinted genes, and chromosome 11p15.5. Pediatr Res 61:43-47.

Squire JA, Li M, Perlikowski S, Fei YL, Bayani J, Zhang ZM and Weksberg R (2000) Alterations of H19 imprinting and IGF2 replication timing are infrequent in Beckwith-Wiedemann syndrome. Genomics 65:234-242.

Taniguchi T, Sullivan MJ, Ogawa O and Reeve AE (1995) Epigenetic changes encompassing the IGF2/H19 locus associated with relaxation of $I G F 2$ imprinting and silencing of H19 in Wilms tumour. Proc Natl Acad Sci USA 92:21592163.

von Kanel T, Gerber D, Schaller A, Baumer A, Wey E, Jackson CB, Gisler FM, Heinimann K and Gallati S (2010) Quantitative 1-step DNA methylation analysis with native genomic DNA as template. Clin Chem 56:1098-1106.

\section{Internet Resources}

Dome JS and Huff V (2011) GeneReviews: Wilms Tumor

Overview. http://www.ncbi.nim.nih.gov/books/NBK1294 (October 12, 2011).

\section{Editor: Marcio C. Silva-Filho}

License information: This is an open-access article distributed under the terms of the Creative Commons Attribution License, which permits unrestricted use, distribution, and reproduction in any medium, provided the original work is properly cited. 